\title{
Palatal Expansion and its Effects in Orthodontics
}

\author{
Aakash Shah*, Purvesh Shah, Santosh Kumar Goje, Romil Shah, Bhumi Modi \\ Department of Orthodontics and Dentofacial Orthopedics, \\ K.M. Shah Dental College and Hospital, Vadodara, Gujarat, India
}

dai: https://doi.org/10.21467/ajgr.2.1.31-36

*Corresponding Author email: aakashshah108@gmail.com

Article History

Received: 14 March 2017

Revised: 10 April 2017

Accepted: 19 April 2017

Published: 21 April 2017

Student(s)

- Aakash Shah

Academic Year: 2016-17

Course Level: Master Degree

Course Name: Master of Dental Surgery (M.D.S.)

Course year: $3^{\text {rd }}$ year

Mentor(s)

- Purvesh Shah

- Santosh Kumar Goje

- Romil Shah

- Bhumi Modi

\begin{abstract}
Palatal expansion has a unique role in dentofacial therapy. The rapid Maxillary expansion which is a type of skeletal expansion involves the opening of the mid-palatal suture and movement of the palatal shelves away from each other. Selection of appropriate appliance should be made by preparing a list of criteria depending on the biomechanical requirements of RME. Rapid expansion affects the maxillary complex, palatal vaults, maxillary teeth, adjacent periodontal structures to get desired expansion in the maxillary arch. Maxillary transverse deficiency in adults is a malocclusion of relatively high prevalence in orthodontics. The rapid maxillary expansion (RME) surgically assisted is considered the preferred procedure to correction of this alteration in patients skeletally mature. This review discusses the types of palatal expansion and skeletal and soft tissue effects of palatal expansion.
\end{abstract}

Keywords: Palatal expansion, Orthodontics

\section{Introduction}

The utilization of palatal expanders by orthodontists began with the father of orthodontics, Edward Angle, and their utilization has been extensively detailed within orthodontic literature [1]. Much of the earliest work on expanders and their indications were conducted by Dr. Andrew Haas. In one of his earliest publications, Haas advocated for the use of palatal expanders in five clinical situations, and although not entirely true today, several elements of this article published over 40 years ago are still highly relevant [2]. Interestingly, as Haas pointed out, the use of palatal expansion as indicated for Class III malocclusions is common, and other prominent authors, such as McNamara, have relayed similar benefits of palatal expansion for Class III correction [3]. Indeed, the use of expanders as a treatment modality is still commonly used today primarily to aid with the correction of crossbites, crowding, and transverse maxillary deficiencies [4]. 


\section{Types of Palatal Expansion}

Despite this relatively common use of expansion as a treatment method, multiple modalities and appliance designs exist. These modalities can be categorized by three primary types of expansion: rapid maxillary expansion, slow maxillary expansion, and surgically assisted maxillary expansion [5]. Furthermore, four types of expanders exist when categorized by activation protocol. These four groups are screw-type, spring-type, magnetic, and Shape Memory Alloy (SMA) activation.

\subsection{Screw-type Expanders}

The screw-type expander category consists of expanders in which manual rotation via a wrench or "key" by either the clinician or patient results in widening of the jack- screw. This design has a well understood and historically common mechanical concept of expansion via turning of a screw-jack. Simply, the amount of screw rotation directly corresponds to the amount of expansion. An advantage of this category includes the flexibility provided to the practitioner to prescribe a certain amount of expansion over a certain period of time. Also, appliances can be adapted to fit a variety of palate sizes and shapes, seemingly limited only by the size and placement of the jackscrew component. However, several drawbacks to the jackscrew appliance do exist. Foremost among these is that upon activation of the screw, a sudden, rapid increase in force is produced which has been shown to possibly result in less physiologic expansion of the palatal suture. Also, this method of expansion places a large responsibility on the patient to comply with expansion protocols, which is a significant disadvantage of this design. Within this category of appliances is the most commonly used Hyrax appliance design, named for its trademarked Hyrax jackscrew [6].

\subsection{Spring-type Expanders}

Spring-type expanders are defined as any appliance that functions via mechanical deformation of a body. This deformation subsequently results in elastic restoration forces that are exerted on the palate and thus results in expansion. This design offers certain advantages, such as less dependency upon the patient to manually activate the expander at a regular interval. Furthermore, theoretically it applies a constant force over a period of time and avoids sudden increases in force as seen in the screw appliances. This predictable amount of force likely results in greater comfort for the patient following initial delivery. The amount of force produced is inversely proportional to the amount of expansion. Therefore, the more expansion produced the less force for further expansion remains and the orthodontist may have to remove and re-activate the appliance if more expansion is needed following treatment. Patient safety is also another concern, as the appliance is delivered "active". Perhaps the greatest drawback of this design is that although springs provide predictable transverse forces, any deformation of the appliance may result in unwanted force in all other planes of space [6].

\subsection{Magnetic Expanders}

Magnetic expanders also exist, and have been referred to as Magnetic Expansion Devices (MED). The goal of magnetic expansion devices is to provide continuous forces of a lesser magnitude than traditional expansion devices. The magnets are applied so that their directionality opposes each other, thus creating a repulsive and expansive force [6]. Theoretically, this would result in a more biologically friendly and less traumatic stimulation of maxillary suture growth, similar to spring type appliances. A study by Darendeliler [7] showed the greatest degree of skeletal expansion occurred in banded appliances with four magnets. A set of two magnets were placed apically to the central and lateral incisors and the second set placed between the second bicuspid and first molar. Although the sample size was small, they did show that MEDs provide a relatively effective method of palatal expansion, which did not rely on patient compliance. Advantages and disadvantages of MEDs are similar to those of the spring type of appliances. However, one significant advantage of MEDs is that they are less prone to deformation and thus have less risk to produce undesirable and unpredictable forces in dimensions other than the transverse. Similar 
Shah et al., Adv.J. Grad. Res.; Vol. 2 Issue 1, pp: 31-36, July 2017

to the spring type, magnetic forces decrease with increasing expansion and therefore also require adjustments like its spring counterparts. This problem can be avoided by placement of magnets of greater strength; however, this may result in less patient comfort and less physiologic suture opening [6].

\subsection{Shape Memory Alloy Appliances}

Finally, the fourth category of expanders, shape memory alloy appliances, utilize the properties of nickel titanium wires and are therefore dependent upon the properties of the shape memory alloy incorporated into the device for expansion. Orthodontists have become increasingly familiar with the properties of Ni$\mathrm{Ti}$ wires and therefore it is prudent to assume their incorporation into expansion devices is a natural progression. However, the drawbacks of a conventional spring appliance still exist with these appliances. Of primary concern is that any deformation of the appliance may result in uncontrolled forces transverse to the direction of expansion. More recently, promising hybrid devices have been constructed to include both Ni-Ti wires and expansion jackscrews; however these appliances re-introduce the major disadvantage of patient compliance [6].

As previously mentioned, palatal expanders can also be categorized based on rate of expansion into three broad categories: rapid, slow, and surgically assisted.

\subsection{Rapid Palatal Expansion}

Rapid palatal expansion (RPE) typically involves two turns per day of a jackscrew expander appliance, commonly a rate of $0.5 \mathrm{~mm}$ expansion per day. Indications for rapid palatal expansion include transverse discrepancies of greater than $4 \mathrm{~mm}$ with dental compensation via buccally tipped maxillary molars, disruption of sutures to aid Class III correction, and moderate maxillary crowding. RPE is contraindicated when there is recession of the alveolar bone on maxillary molars or premolars, presence of anterior open bite, high mandibular plane angle, convex profile and doubtful patient compliance [5]. Furthermore, RPE is contraindicated in mature patients beyond the growth spurts, however many practitioners may still choose this treatment modality on older patients. Clinically, patients are instructed to turn the expander once in the morning and once in the evening, or only once a day, commonly for a period of two to three weeks followed by a retention period of at least three months. This rapid rate of activation and relatively large force application is thought to maximize orthopedic skeletal expansion while minimizing dental movements [5]. RPEs can be designed as banded or bonded appliances, and can be tooth-borne, tissueborne, or tooth and tissue borne. Some examples of rapid palatal expanders are the Hyrax expander, Issacson expander, and Haas expander [5].

\subsection{Slow Palatal Expansion}

Slow palatal expansion (SPE) is a process by which light, relatively continuous force levels commonly in the range of 450-900 grams are applied. It is thought that the lighter forces result in less resistance from sutural structures, thus allowing more bone formation and activity in the intermaxillary suture, but possibly less suture opening. Furthermore, post-expansion stability and retention may be greater with SPE. Appliance designs vary greatly for SPE, with some examples being the Quadhelix, the Coffin appliance, magnet expanders, W-arches, and spring jets. The hyrax expander can also be used for SPE, with a maximum of $1 \mathrm{~mm}$ expansion per week applied.

\subsection{Surgically Assisted Palatal Expansion}

Finally, surgically assisted palatal expansion occurs when expansion is aided with surgical intervention. Surgical expansion involves either surgically assisted rapid palatal expansion (SARPE) or segmental maxillary surgery such as a Lefort osteotomy. Indeed, this procedure allows for expansion beyond skeletal maturation but is highly invasive and complex [5].

Rapid Maxillary expansion occupies unique niche in orthodontic treatment. Maxilla will grow in small amount transversely throughout the life. When palatal expanders are used, widening of the maxillary arch may occur via either orthodontic or orthopedic movements, or a combination of both. Generally, the 
Palatal Expansion and its Effects in Orthodontics

initial increase in width occurs as teeth compress and stretch the periodontal tissue. This is an orthodontic movement as the teeth are tipped laterally due to sudden force application, and studies have shown that this gain is usually completed within one week $[8,9]$. Compressive forces continue as long as the expander is active. These forces result in resorption of the buccal alveolar plate at the interface of the tooth root and periodontal attachment, resulting in further orthodontic tooth movement. This movement is bodily movement of the teeth into a wider arch form. Orthopedic expansion of the maxilla occurs via separation of the midpalatal suture. This separation occurs when forces applied exceed the bioelastic tensile strength of the elements within the suture, or the interdigitation of the bony spicules. As long as the transverse force of expansion is greater than this tensile strength, further separation of the suture occurs. Once the expander is locked, the suture reorganizes [10].

\section{Skeletal Effects of Palatal Expansion}

Palatal expansion, similarly to several treatment modalities in orthodontics, can produce profound changes in a patient's overall facial appearance. Cephalometric studies have historically been used to describe these changes. In one of the earliest studies conducted by Haas, he found that cephalometric analysis revealed an increased internasal width following expansion, concluding that there is "coincidental widening of the nose and lowering of its floor". Of course, these studies focused solely on skeletal changes via radiographic appearance. He also found that there was a widening of the mandibular arch, and postulated that this may be attributed to tongue pressure from inferior displacement by the expansion appliance [11]. White examined pretreatment and post treatment records of thirty patients undergoing palatal expansion. He noted clockwise movement of the mandible following expansion treatment [12]. Also of note, however, was that he noticed significant widening of the internasal width on posteroanterior cephalograms. Chung and Font examined pre and post-treatment records on twenty children who were treated with Haas type expanders. The authors also found statistically significant forward and downward displacement of the maxilla, and clockwise rotation of the mandible following expansion treatment. Furthermore, significant facial changes beyond the maxilla and mandible were noted. Anterior facial height and the width of both the maxilla and the nasal cavity increased significantly [13].

The advancement of cone beam computed tomographic (CBCT) radiology has allowed orthodontists and researchers to explore changes produced by palatal expansion more precisely. Habeeb evaluated pre and post-treatment CBCT images of twenty eight patients who were treated with bonded Haas expander appliances. The investigator found that both ANS and PNS moved significantly downward from expansion treatment. In addition, they found that while the maxilla was anteriorly displaced, the maxillary central incisors moved posteriorly from their initial position [14]. A similar CBCT study was conducted using thirty patients, mean age of $13.8+/-1.7$ years, who underwent palatal expansion with a banded Hyrax appliance design. High-resolution CBCT images were taken before and after expansion, which the authors claimed allowed for more accuracy than previous studies. They found that rapid palatal expansion resulted in an increase in nasal width which was statistically significant $(\mathrm{p}<.0001)$. On average, nasal width increased by $1.89 \mathrm{~mm}$ [15]. It may be important to note that this change was due primarily to expansion, independent of aging [15]. Similar findings occurred in a CBCT study on fourteen children who underwent rapid palatal expansion. All patients were treated with a four-banded Hyrax appliance design and instructed to activate the appliance 1 turn/day for 28 days or until complete correction of pre-existing crossbites. CBCT scans were taken prior to initiation of treatment and again 3 to 4 months following expansion. From their data, the authors agreed with previous studies that RPE indeed may cause significant expansion of the maxilla. Furthermore, they concluded that there is a significant increase in the cross-sectional area of the upper airway following rapid maxillary expansion [16]. Therefore, recent CBCT studies show agreement with earlier hard tissue studies on expansion effects.

\section{Soft Tissue Effects of Palatal Expansion}

Although the studies listed above discuss treatment outcomes that altered facial characteristics of 
Shah et al., Adv.J. Grad. Res.; Vol. 2 Issue 1, pp: 31-36, July 2017

orthodontic patients, it is important to our work to also investigate studies which examine effects of expansion on soft tissues. Mota dos Santos evaluated twenty patients who underwent rapid palatal expansion. Lateral cephalograms were taken prior to expansion treatment, immediately following expansion treatment, and finally after a period of retention. Any conclusions on long-term soft tissue changes based on their data were inconclusive. The authors did note statistically significant changes in the following cephalometric measurements: S-li, H Line-Prn, E Line-Li, E Line-Ls, ANS'-Me'. They concluded these changes were primarily due to the presence of the expander appliance. However, they concluded that following a retention period of six months, minimal soft tissue changes are seen following expansion [17]. A similar study was conducted which consisted of 18 patients with bilateral posterior crossbite. All patients underwent rapid palatal expansion using bonded Hyrax expanders, the same appliance used in the previous study. Cephalometric radiographs were again taken at three separate time points, prior to treatment, following completion of expansion, and retention (mean 5.95 +/- 0.35 months). The results were slightly different than those of Mota dos Santo's study, as soft tissue facial angle decreased $(\mathrm{P}<0.05)$, and $\mathrm{H}$ angle and skeletal profile convexity significantly increased $(\mathrm{P}<0.001)$ following expansion. Also, the authors noted that while some relapse did occur, both $\mathrm{H}$ angle and profile convexity remained significantly altered following retention periods [18]. In summary, the literature database supports the notion that rapid palatal expansion produces soft tissue changes.

\section{Conclusions}

RME is a technique of choice in several cases like moderate to severe transverse discrepancy. This helps us to increase the arch length in moderate crowding cases where arch length is deficient. Orthopedic transverse correction can be done by tipping of posterior teeth buccally along with separation of midpalatal suture. Overcorrection is required to compensate the relapse. Nowadays, CBCT has being used to assess the accurate three dimensional orthopedic changes after RME treatment. Rapid maxillary expansion is an orthopedic movement that mainly puts a strain on the maxillary suture (but other sutures are also affected), the maxillary bones, the teeth and the periodontium. Particular care must be taken for the gum (width and thickness) when expansion (regardless of the method used) is planned. Adults show more gingival recession problems and expansion may have a negative effect on the gum and the periodontium if it is not planned or executed properly. Advances in orthopedic expansion by surgical method include SARPE and segmental maxillary surgery which are simple, safe, noninvasive and similar to RME. Satisfactory occlusion can be established by subsequent, comprehensive orthodontic treatment with good stability. It is useful for cleft palate patients who often have an unpredictable blood supply system.

\section{How to cite this article:}

Shah, A., Shah, P., Goje, S., Shah, R., \& Modi, B. (2017). Palatal Expansion and its Effects in Orthodontics. Advanced Journal Of Graduate Research, 2(1), 31-36. doi: https://doi.org/10.21467/ajgr.2.1.31-36

\section{References}

[1] Angell EH. Treatment of irregularity of the permanent or adult teeth. Dent Cosmos. 1860 May;1(10):540-4.

[2] Haas AJ. Palatal expansion: just the beginning of dentofacial orthopedics. American journal of orthodontics. 1970 Mar 1;57(3):21955.

[3] McNamara Jr JA. An orthopedic approach to the treatment of Class III malocclusion in young patients. Journal of clinical orthodontics: JCO. 1987 Sep;21(9):598-608.

[4] Proffit WR, Fields HW, Sarver DM. Contemporary Orthodontics. 4th ed. St. Louis, MO: Mosby; 2007.

[5] Agarwal A, Mathur R. Maxillary expansion. International Journal of Clinical Pediatric Dentistry. 2010 Sep;3(3):139.

[6] Romanyk DL, Lagravere MO, Toogood RW, Major PW, Carey JP. Review of maxillary expansion appliance activation methods: engineering and clinical perspectives. Journal of dental biomechanics. 2010;2010.

[7] Darendeliler MA, Strahm C, Joho JP. Light maxillary expansion forces with the magnetic expansion device. A preliminary investigation. The European Journal of Orthodontics. 1994 Dec 1;16(6):479-90.

[8] Cotton LA. Slow maxillary expansion: skeletal versus dental response to low magnitude force in Macaca mulatta. American Journal of Orthodontics. 1978 Jan 1;73(1):1-23. 
Palatal Expansion and its Effects in Orthodontics

[9] Hicks EP. Slow maxillary expansion: a clinical study of the skeletal versus dental response to low-magnitude force. American journal of orthodontics. 1978 Feb 1;73(2):121-41.

[10] Bell RA. A review of maxillary expansion in relation to rate of expansion and patient's age. American journal of orthodontics. 1982 Jan 1;81(1):32-7.

[11] Haas AJ. Rapid expansion of the maxillary dental arch and nasal cavity by opening the midpalatal suture. The Angle Orthodontist. 1961 Apr;31(2):73-90.

[12] White RE. A cephalometric appraisal of changes in the maxillofacial complex resulting from palatal suture expansion utilizing fixed appliance therapy. American Journal of Orthodontics. 1972 May 31;61(5):527-8.

[13] Chung CH, Font B. Skeletal and dental changes in the sagittal, vertical, and transverse dimensions after rapid palatal expansion. American journal of orthodontics and dentofacial orthopedics. 2004 Nov 30;126(5):569-75.

[14] Habeeb M, Boucher N, Chung CH. Effects of rapid palatal expansion on the sagittal and vertical dimensions of the maxilla: a study on cephalograms derived from cone-beam computed tomography. American Journal of Orthodontics and Dentofacial Orthopedics. 2013 Sep 30;144(3):398-403.

[15] Garrett BJ, Caruso JM, Rungcharassaeng K, Farrage JR, Kim JS, Taylor GD. Skeletal effects to the maxilla after rapid maxillary expansion assessed with cone-beam computed tomography. American Journal of Orthodontics and Dentofacial Orthopedics. 2008 Jul 31;134(1):8-e1.

[16] Chang Y, Koenig LJ, Pruszynski JE, Bradley TG, Bosio JA, Liu D. Dimensional changes of upper airway after rapid maxillary expansion: a prospective cone-beam computed tomography study. American Journal of Orthodontics and Dentofacial Orthopedics. 2013 Apr 30;143(4):462-70.

[17] dos Santos BM, Stuani AS, Stuani AS, Faria G, Quintão CC, Stuani MB. Soft tissue profile changes after rapid maxillary expansion with a bonded expander. The European Journal of Orthodontics. 2012 Jun 1;34(3):367-73.

[18] Kiliç N, Oktay H. Effects of rapid maxillary expansion on nasal breathing and some naso-respiratory and breathing problems in growing children: a literature review. International journal of pediatric otorhinolaryngology. 2008 Nov 30;72(11):1595-601.

\section{Publish your research article in AIJR journals-}

$\checkmark$ Online Submission and Tracking

$\checkmark$ Peer Reviewed

$\checkmark$ Rapid decision

$\checkmark$ Immediate Publication after acceptance

$\checkmark$ Open Access (Articles freely available online)

$\checkmark$ Retain full copyright of your article.

Submit your article at journals.aijr.in 